\title{
Interactive comment on "Mixing State of Refractory Black Carbon of the North China Plain Regional Aerosol Combining a Single Particle Soot Photometer and a Volatility Tandem Differential Mobility Analyzer” by Yuxuan Zhang et al.
}

Anonymous Referee \#1

Received and published: 19 June 2017

Summary: This paper discusses investigations of the SP2 instrument for measuring black carbon, and its comparison with a differential mobility analyzer (particle sizer) with volatility applied. The technical work appears to be competent. The interpretation, however, appears somewhat rambling. Additionally, these authors published an earlier paper (Atmos Meas Techniques) that investigated many similar issues including measurements from a site near Beijing (Xianghe). I think this paper could be published, but the presentation needs to be sharpened quite a bit. The authors need to explain exactly what questions are being asked, how they are relevant to atmospheric chem-

Printer-friendly version

Discussion paper 
istry and radiative transfer, how the findings in this paper provide new information, and summarize recommendations. Especially compared with their earlier paper, the authors could state what questions had been left open, and what the present paper now addresses.

The writing here is not bad. But it needs to be reviewed by a native English speaker. Examples in the introduction: "results in largest uncertainties" (line 15), "while the rest components" (line 22), "The causes are due to that" (line 25). I won't list all of them. In particular "inversed" cannot be used as a verb as it is here, but instead one should say "determined by inversion. "

I also note that I wrote this review before looking at the comments of the other reviewer because they should be independent. There could be some disagreement or repetition.

Introduction lines 8-10. Most of these citations discussed absorption, not even climate and certainly not BC as second most important warming agent. They do not support the statement.

mixing state: authors should state what "mixing state" means. On page 3 line 19, they say that it was quantified. The reader should know what to look for. Especially the use of "externally-mixed BC particles" throughout the manuscript is awkward. There could be externally-mixed aerosol populations in which bare BC exists, but when counting the bare BC particles only, one wouldn't say these individual particles are "externally mixed."

equations $3 \& 4$. These are not real equations. They are dependences, I think. In that case it should just be stated: the determination of the optical diameter depends on the scattering cross-section and the assumed refractive index, rather than writing as a false equation. Just before this equation, authors state that equations 3 and 4 list input parameters for the Mie model. But scattering cross section is an output from a Mie model, not an input. 
Fig 3 compares the mobility-determined size with the size determined from the SP2 scattering cross section. But the refractive index used in this determination was already fit, that is, we should expect the two to agree because one was derived from another. I don't think this justifies anything.

Line 15 in section 3 says, 'The agreement revealed that the LEO method was applicable for ambient aerosol particles." The authors haven't discussed the LEO method enough to enable the reader to evaluate this statement. If the LEO method were valid, what would we see? If it were invalid, what would we see or not see?

Line 15 also: "It also validated the assumption of a spherical BC particle in the Mie model calculations." I don't think it does anything of the kind. It says that the BC in the particle perhaps doesn't change the scattering very much whether it is spherical or non-spherical.

Figure 4 does not show the fractal dimension of the particles, but they are inferred from the data shown in figure 4.

Section 3.1.2. I agree that the density of a bare BC particle (aggregate) should be much less than the density of the void-free particle. I don't think anyone has ever suggested that it would have the density of the void-free particle. I am wondering what is the relevance of the density given here $(0.25-0.45 \mathrm{~g} / \mathrm{cm} 3)$. Where would it be used.

Section 3.1.3. I am confused by this section. 1. Some of this information, I think should be in the Methods section. 2. Again there is an assessment of the LEO-fit without too much explanation. It would be useful to see what reader should expect compared to what is found. 3. "... which demonstrated the potential of optical sizing instruments... in determining the bipolar charge distribution of aerosol particles." Since the theoretical distribution seems to work fine, why do we need the optical sizing instrument? It seems like the authors are just looking for reasons to use the SP2 that are not needed.

Section 3.2. (comparison VTDMA/SP2) The finding of refractory, non-BC material in 
the small particles could be important. But the SP2 has difficulty measuring the small particles (or maybe just different efficiency) because of low scattering. How was this accounted?

Section 3.3. This discussion equates aging with coating thickness to find that BC containing particles are "more aged" and then associates these with long-range transport. Typically studies refer to some kind of photochemical age (independent measure of aging). It hasn't really been proven here that these particles have been in the atmosphere longer since emission than particles without thick coating. Authors should be clear that their statements are inferences and not proven.

thick coating: Authors should be clear about what they mean by "thick coating." I could not find a definition. They identify coatings as thick and then say this will lead to lensing and enhanced absorption. But a $\times 3$ increase in mass due to coating is only $40 \%$ increase in radius and $30-40 \%$ increase in absorption. Claims about strong enhancement, would be better if some physical basis were discussed. I also think the number of uncoated particles (what's called 'externally mixed' in the paper) should be shown here. Fig 9 suggests that the bare particles would actually be the largest fraction.

Figure 10 \& figure 11. I don't understand a discrepancy here. Figure 10 shows (as stated in the text) that the mode of $\mathrm{Dp} / \mathrm{Dc}$ (particle to core) ratio is 2-3 and many of the ratios are greater than 3. But I don't see particles with such large Dp/Dc in figure 11 . $\mathrm{Dp} / \mathrm{Dc}=3$ means that coating thickness is $2 x$ core radius. Fig 11 shows that hardly any coating thicknesses are $2 x$ greater than the core radius. Anyway I think that fig 11 would be easier to understand if radius were on the $x$ axis.

Figure 11 description, "The CT of internally-mixed BC particles decreased as Dc increased... " Isn't this required from the way the figure was made? The DMA selects one diameter. That diameter is just the core + coating.

The final statement, about "importance of $\mathrm{BC}$ aerosols in polluted regions in China to climate change", is not supported. Perhaps it is true, but although the absorption 
may be a little stronger (per mass) than in developed countries, it does not make the aerosols hugely more important. Furthermore, no inferences about climate change were calculated, such as radiative forcing.

Interactive comment on Atmos. Chem. Phys. Discuss., https://doi.org/10.5194/acp-2017-222, 2017. 